Proc $2^{\text {nd }}$ APCRS

\title{
Effects of modifying fin-kick behavior on rates of damage to reef environments by scuba divers
}

\author{
Yeng SU', Chun-Chun TSAI ${ }^{2}$, and Tsen-Chien $\mathrm{CHEN}^{3, *}$
}

${ }^{1}$ Department of Marine Sports and Recreation, National Penghu University, Republic of China

${ }^{2}$ Department of Leisure \& Recreation Studies, Matou Campus, Aletheia University, Republic of China

${ }^{3}$ Department of Leisure Management, Ming Hsin University of Science and Technology, Sinfong, Hsinchu 304, Taiwan, Republic of China

* Corresponding author: Tsen-Chien CHEN

E-mail: blennidae@yahoo.com.tw

\begin{abstract}
Coral reefs continue to attract increasing numbers of scuba divers, leading to concerns about diver's impacts on reefs. Among various types of impacts, fin kicks damage the corals reefs the most. To reduce fin-kick accidents, a technique to keep divers' fins from hitting reefs during diving was evaluated. In this study, lifting diver's fins higher than usual by shifting the diver's center of gravity towards the upper body was carried out. Three experimental conditions for the center of gravity were respectively set up by positioning lead blocks, in combination with wearing a weight belt around the waist or chest, to examine differences in rates of fin kicks to reefs. One weight belt was placed at the waist in the first experiment, at the waist and chest in the second, and at the chest in the third for arranging the low, the middle, and high center of gravity respectively. There were significant differences found in the rates of fin kicks among the three conditions. The mean rates of fin kicks were 2.56 contacts $10 \mathrm{~min}^{-1}$ in the first experiment, and 0.94 in the second, and 0.06 in the third. The result indicated that fin-kicking on reefs is avoidable by changing diver's center of gravity to the upper body.
\end{abstract}

Keywords SCUBA, diver, tourist impact, coral reefs

\section{Introduction}

Colorful corals, invertebrates, and fishes attract thousands of tourists to visit coral reefs. Scuba diving is a popular recreational activity to support people to get close look at these marine animals. The number of people using SCUBA diving has increased recently. However, excessive concentrations of divers threaten reef health (Zakai and Chadwick-Furman 2002). Divers touch corals due to curiosity. Some photographers deliberately creep over reefs in order to take pictures (Rouphael and Inglis 2001). Divers kick, grab, scrape the corals with their fin blades, or scrape it with their console or tanks, without knowing that harm to the corals has occurred (Harriott et al. 1997). Such contact may directly break the corals or remove the outer layer of the tissue of the coral. Wounded coral tends to become infected by pathogens and has a higher risk of dying (Hall 2001). Among the many types of impacts caused by divers, fin kicks damage corals the most (Zakai and Chadwick-Furman 2002). In this study we have proposed a technique for reducing the effects of fin kicks. It is hoped that the technique recommended herein is helpful for managing divers on coral reefs. 


\section{Material and methods}

By observing scuba divers' behaviors, it was found that many divers are unable to keep their body in a horizontal position in mid-water as they are trained to do, but end up in a feet-down position. This position easily causes fin kicking on coral reefs. If a diver's feet and fins can remain in a higher place than usual during diving, the occurrence of fin kicks can be decreased. In the present study, divers' feet and fins were experimentally lifted up by gradually shifting the diver's center of gravity upwards, and the numbers of fin kicks striking the reefs for different experimental conditions were compared.

Data were collected on 18 firemen trainees responsible for disaster rescue and emergency medical services, who had taken advanced scuba diving courses taught by the first author (Su Y), from 5 to 10 July 2004 in Lienchiang County, Taiwan. Experiments were carried out using three conditions for a diver's center of gravity. First, all trainees were asked to put on a lead-block weight belt around their waist as it is traditionally worn on the first training day (Fig. 1a). Next, trainees tightened the belt with half of the lead blocks around the waist, and the other half around the chest on the second day (Fig. 1b). Finally, they were requested to fasten the belt with all of the lead blocks around the chest on the third day (Fig. 1c). So, a diver's center of gravity gradually shifted toward the upper body in this sequence. Herein, trainees wore the weight belt around the chest in a way different from that around the waist (in the latter case, the belt was tightened outside of the wetsuit and inside the buoyancy compensator device (BCD)), otherwise there might be difficulty with a quick weight dump in case of an accident. Here, a technique of wearing the belt around chest was suggested. Two belts were necessary, one was composed of lead blocks and two easy-to-open buckles engaged on both sides, while the other was a bare belt (Fig. 2). The diver's chest was girdled with the lead-block belt outside of the BCD and the bare belt, which ran through the BCD armhole and around the back side between the wetsuit and the $\mathrm{BCD}$ (Fig. 2). Both belts were fixed by two buckles around the $\mathrm{BCD}$ armhole. Therefore, the lead blocks remained outside of the $\mathrm{BCD}$, and divers could easily drop the weight
(A)

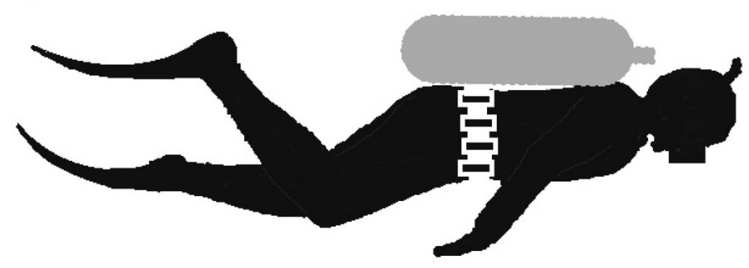

(B)

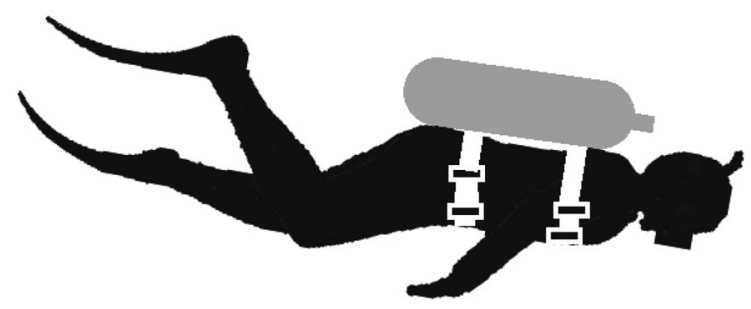

(C)

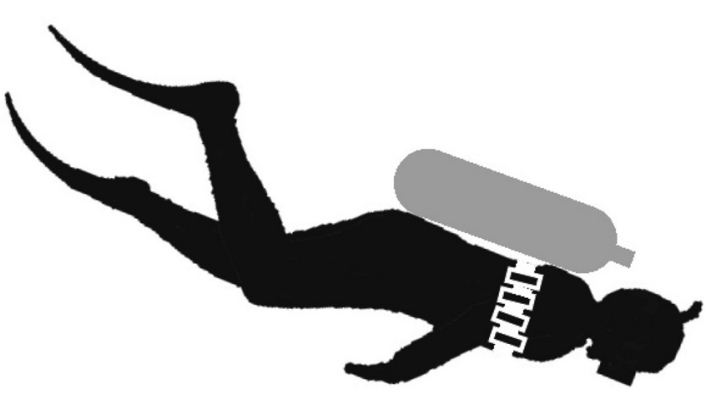

Fig. 1 Divers wearing weight belts with lead blocks in three ways. (a) A diver wearing the belts with all lead blocks around waist, (b) a diver wearing half of the lead blocks around the waist, and the other half around the chest, and (c) a diver wearing the belts with all of the lead blocks around the chest.

belt if there was an accident.

Each time data collection began when a single lesson was finished, and the assistant instructors guided the trainees around the waters for the remainder of the class, and ended at the point when the trainees began their ascent to the water surface. Each trainee was individually marked with number on tank in advance for identification. Data were collected by three observers, and each observer followed one trainee to record data for 10 mins. During the remainder of the class in a single lesson, data of one or two trainees' kicks could be obtained. There were four lessons on each training day. Each observer recorded the same trainees' behaviors till that all of the experimental works were completed. The number of times which a fin contacted either a reef or the sand, was recorded. All 
experiments were carried out on the same group of trainees. Therefore, Friedman's test was used for the statistical analysis.

\section{Results}

All fin kicks against a surface of 18 trainees were recorded (Table 1). There were significant differences in the numbers of fin kicks among the three conditions

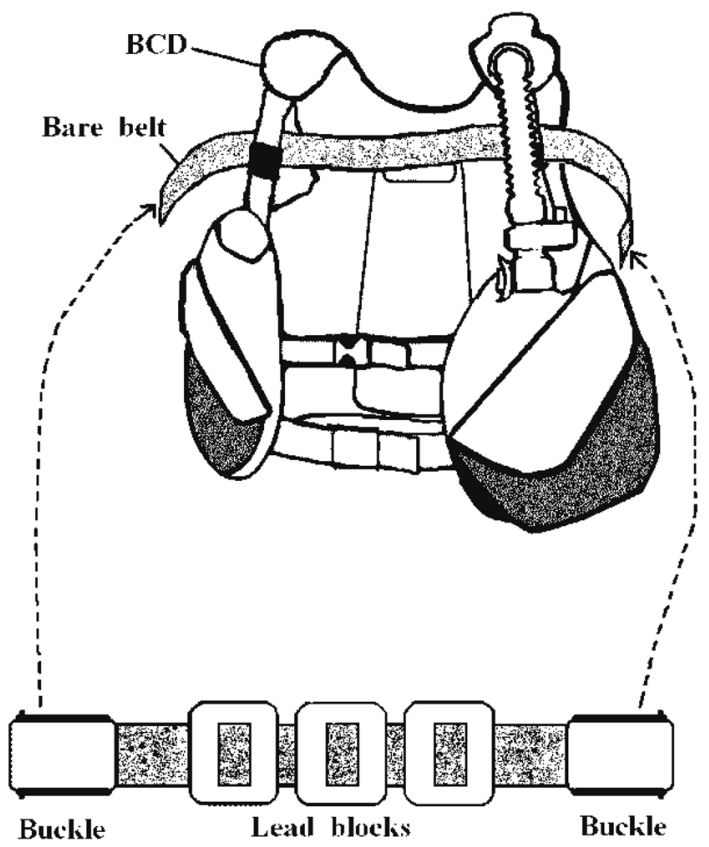

Fig. 2 Diving gear arrangements for wearing the leadblock weight belt around the chest. One bare belt is run through the armholes of the buoyancy compensator device (BCD), another belt is composed of lead blocks and two quick-release buckles engaged on each side. The diver is girdled by the bare belt, which runs through between the wetsuit and the BCD, and around the back, while the leadblock belt runs around the chest outside of the BCD.
(Friedman's test: $\chi 2=31.87, \mathrm{p}<0.01$ ). As a trainee's center of gravity moved upwards on the body (low, middle, and high center of gravity), the mean number of fin kicks showed a decreasing trend (with respective means of 2.56, 0.94 , and 0.06 contacts $10 \mathrm{~min}^{-1}$ ).

\section{Discussion}

The increasing occurrence of diver impacts has raised wide concern. Some attempts have been made to reduce such impacts. For example, accurate information concerning the difficulties facing coral reefs must be translated into practice by instructors who need to help their students and customers become more environmentally friendly reef users (Medio et al. 1997; Barker and Roberts 2004). In this study, we aimed to change diver's fin-kick behaviors. After shifting a diver's center of gravity upwards, the numbers of fin kicks indeed decreased. Once a diver had the weight around the chest, the incidences of fin kicks were almost totally eliminated. In comparison to the traditional attire that divers wear in a horizontal body position during diving when using this technique, the feet of divers are involuntarily slightly tilted up. The extra space between the diver's fins and reefs prevented corals and associated organisms from being contacted. If this idea is widely accepted and used for saving corals, we believe that corals could be saved. Weight-integrated$\mathrm{BCD}$, commonly available in dive shop. Because all of the lead pouches are placed higher than waist, some smaller pouches of lead can be located on around the shoulders. Diver's center of gravity by using weight-integrated-BCD is higher than using traditional $\mathrm{BCD}$ and waist weight belt.

Table 1 Number of kicking of each experiment and average.

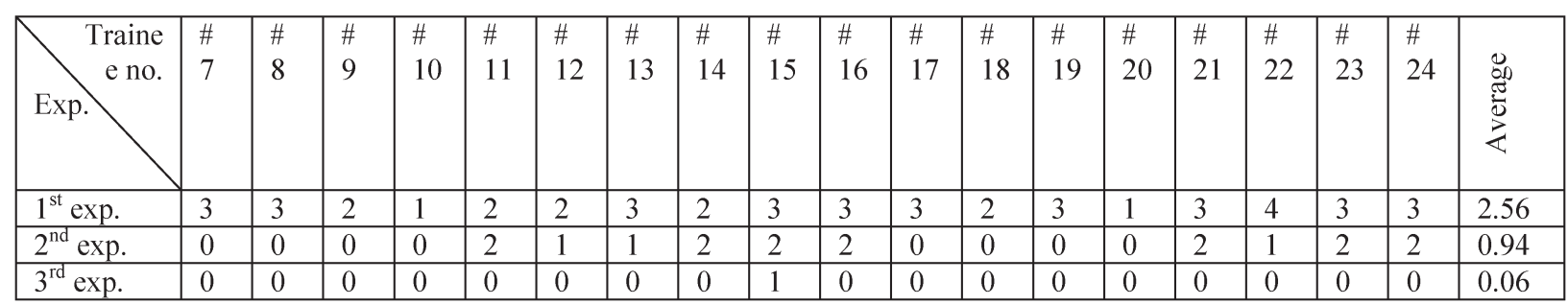




\section{Acknowledgements}

We would like to thank Kuo SC for his Help in editing the figures and Chen $\mathrm{CW}$ for carrying out field works.

\section{References}

Barker NHL, Roberts CM (2004) Scuba diver behaviour and the management of diving impacts on coral reefs. Biol Conserv 120: 481-489

Hall VR (2001) The response of Acropora hyacinthus and Montipora tuberculosa to three different types of colony damage: scraping injury, tissue mortality and breakage. J
Exp Mar Biol Ecol 264: 209-223

Harriott VJ, Davis D, Banks SA (1997) Recreational diving and its impact on marine protected areas in eastern Australia. Ambios 26: 173-179

Medio D, Ormond RFG, Pearson M (1997) Effect of briefings on rates of damage to corals by SCUBA divers. Biol Conserv 79: 91-95

Rouphael AB, Inglis GJ (2001) "Take only photographs and leave only footprints"?: an experimental study of the impacts of underwater photographers on coral reef dive sites. Biol Conserv 100: 281-287

Zakai D, Chadwick-Furman NE (2002) Impacts of intensive recreational diving on reef corals at Eilat, northern Red Sea. Biol Conserv 105: 179-187

C Japanese Coral Reef Society 